\title{
p63 expression in assessment of bronchioloalveolar proliferations of the lung
}

\author{
Hina A Sheikh, Kimberly Fuhrer, Kathleen Cieply and Samuel Yousem \\ Department of Pathology, University of Pittsburgh Medical Center, Pittsburgh, PA, USA
}

\begin{abstract}
Discrimination of well-differentiated pulmonary adenocarcinoma from reactive bronchioloalveolar epithelium can be difficult on routine histology, especially with small biopsies. Ancillary studies to help in this distinction are desirable. p63, a p53-homologous nuclear protein, is a marker of reserve cells of the bronchus and terminal lobular unit. In this study, 33 cases of adenocarcinoma (20 open lung and 13 transbronchial/percutaneous biopsies) and 43 cases of benign lungs with fibrosis and metaplasia (22 open lung and 21 transbronchial/ percutaneous biopsies) were studied for nuclear p63 expression by immunohistochemistry (Dako, Carpinteria, CA, USA). Five additional cases each of atypical adenomatous hyperplasia and adenosquamous carcinoma and three cases of squamous carcinoma (all open lung biopsies) were also stained. The diagnostic categories of benign lung conditions were usual interstitial pneumonia, parenchymal scar, cryptogenic organizing pneumonia and diffuse alveolar damage. In neoplastic cases, p63 positivity was calculated as percentage of all tumor cells examined. In areas of normal lung, p63 decorated the reserve cells of large and small airways and occasional cells of the distal lobular unit. In fibrotic reactive processes, an interrupted but distinct pattern of nuclear staining was present in all cases, with staining of basal cells of the airways as well as bronchiolar- and squamous-metaplastic epithelium $(43 / 43,100 \%)$. p63 immunoreactivity was less uniform in areas of acute lung injury within these cases. One adenocarcinoma and two cases of atypical adenomatous hyperplasia showed strong immunoreactivity ( $>\mathbf{8 0} \%$ ), while three adenocarcinomas highlighted only rare tumor nuclei $(<5 \%$ of tumor cells). Morphologic areas where p63 immunostaining was not helpful included the junction of normal lung and lepidic growth of adenocarcinoma, and retrograde spread of adenocarcinoma into small airways. Our results highlight the differential expression of p63 across various bronchioloalveolar lesions. Moreover, p63 may be helpful in distinguishing reactive from neoplastic glandular proliferations in the lung.
\end{abstract}

Modern Pathology (2004) 17, 1134-1140, advance online publication, 18 June 2004; doi:10.1038/modpathol.3800163

Keywords: p63; p53; bronchial reserve cells; bronchioloalveolar; adenocarcinoma

Biopsies of peripheral pulmonary adenocarcinomas obtained by transbronchial or percutaneous routes are small. Correct histologic diagnosis on such small samples often depends heavily on clinical, radiological and bronchoscopic correlation. Even with appropriate sampling and clinical and radiologic information, the distinction between noninvasive bronchioloalveolar adenocarcinoma and reactive and/or adenomatous hyperplasias is difficult, especially when there is minimal atypia present. ${ }^{1,2}$ Similarly, benign pulmonary epithelium entrapped within pulmonary scars, may be confused with invasive well-differentiated adenocarcinoma in a desmoplastic stroma.

Correspondence: Dr HA Sheikh, MD, Department of Pathology, A-610 Scaife Hall, 200 Lothrop Street, Pittsburgh, PA 15213, USA. E-mail: sheikhha@msx.upmc.edu

This study was presented at The College of American Pathologists' meeting in San Diego, in September 2003.

Received 11 December 2003; revised and accepted 1 April 2004; published online 18 June 2004
DNA ploidy status, morphometric analysis, and p53 expression ${ }^{3-5}$ have been used to separate welldifferentiated adenocarcinomas from atypical adenomatous hyperplasia. These ancillary techniques, while useful in understanding progression of pulmonary adenocarcinoma, are cumbersome and often show continuous variation from reactive to neoplastic conditions. Hence, there is a need for simpler ancillary studies that can be used in the day-to-day practice of diagnostic pathology and that reliably differentiate reactive and neoplastic proliferations of the bronchioloalveolar epithelium.

p63 is a p53 homologous nuclear protein, that is expressed in basal cells of stratified squamous and glandular epithelia. ${ }^{6}$ It has recently found application in prostate needle biopsies, where its sensitivity as a basal cell marker is equal to or greater than that of high molecular weight cytokeratin..$^{7-9}$ In the lung, it has been mainly studied in different histologic subtypes of epithelial neoplasms, with the highest expression consistently noted in squamous cell carcinomas. ${ }^{10,11}$ The pattern of staining in squamous 
carcinomas is also unique, with outer 'basilar' cells showing greater nuclear immunoreactivity compared to inner differentiated 'terminal' cells. ${ }^{11}$ The frequency of expression in pulmonary adenocarcinomas is lower, with most cases showing only focal staining. Neuroendocrine carcinomas are nonimmunoreactive for the most part, with the exception of some large-cell carcinomas. Interestingly, adenosquamous carcinomas are almost always positive, with immunoreactivity confined to squamoid areas, with one study describing staining of basilar cells in the glandular component as well. ${ }^{11}$

While p63 expression in normal lung has been described, its expression in benign lung processes with reactive epithelial hyperplasia and metaplasia has not been examined. With the success of p63 as a basal cell marker in the prostrate, and the presence of p63-immunoreactive basal cells in the proximal portion of the normal bronchioloalveolar tree, we compared expression of this nuclear protein in pulmonary adenocarcinomas, atypical adenomatous hyperplasia and a variety of acute, subacute and chronic reactive lung conditions, in order to assess the usefulness of this stain in the differential diagnosis of glandular proliferations encountered in open lung biopsies and in smaller diagnostically challenging transbronchial/percutaneous lung biopsies.

\section{Materials and methods}

A total of 94 archival routinely processed, formalinfixed paraffin-embedded surgical pathology specimens from bronchoscopic and transthoracic biopsy, wedge resection, lobectomy and pneumonectomy specimens were examined. The tumors studied included primary pulmonary adeno-, adenosquamous and squamous cell carcinomas. Five cases of atypical adenomatous hyperplasia encountered incidentally in tumor resection specimens were also studied. Diagnostic categories of benign lung conditions included zones of honeycomb change in usual interstitial pneumonia, localized pulmonary scars (apical caps), cryptogenic organizing pneumonia and diffuse alveolar damage.

Immunohistochemical staining for p63 was performed as follows: $4-\mu \mathrm{m}$ thick sections were deparaffinized, treated with $3 \%$ hydrogen peroxide to block endogenous peroxidase activity and then treated with $0.01-0.11 \mathrm{M}$ citric acid $(\mathrm{pH}$ 6.0) for $10 \mathrm{~min}$ at $100^{\circ} \mathrm{C}$ for antigen retrieval. Slides were then incubated with anti-p63 monoclonal antibody at a dilution of 1:300 (M7247, Dako, Carpinteria, CA, USA) and stained using the streptavidin-biotin kit and diaminobenzidine as chromogen. Hematoxylin was used for counterstaining.

p63 staining was considered positive only if distinct nuclear staining was present. Sections from nonstaining tumors were excluded in the absence of positive staining of normal bronchial reserve cell nuclei that served as internal positive control.

Slides were reviewed independently by two pathologists and cases where disagreement existed were resolved by 'double scoping'. In pulmonary neoplasms, p63 positivity was scored as a percentage of all tumor cells observed in the slide section. In cases of benign lung disease, an attempt was made at identifying areas of increased p63 nuclear staining in the bronchioloalveloar tree.

Three cases of usual interstitial pneumonia and five cases of pulmonary adenocarcinoma (all open lung biopsies) were excluded due to nonspecific cytoplasmic staining of tumor cells, macrophages and lymphocytes. Two additional cases, one each of transbronchial and percutaneous biopsy, of benign fibrotic lungs were excluded due to nonstaining of normal bronchial reserve cells (internal control) present in the tissue section.

\section{Results}

In areas of normal lung histology, p63 decorated the basal reserve cells of large and small airways and occasional cells of the distal lobular unit (Figure 1a). Ciliated cells, goblet cells and alveolar epithelial cells did not stain.

All cases of acute/subacute lung conditions showed nuclear immunoreactivity of reserve cells of the bronchial epithelium and variable immunoreactivity around fibroblastic foci and organizing pneumonia (10/10, 100\%, Figure 1b). Areas of acute lung injury (acute diffuse alveolar damage, acute fibrinous pneumonia) within these cases were uniformly nonimmunoreactive.

All cases of fibrotic, reactive processes showed an interrupted but distinct pattern of nuclear staining in zones of entrapped bronchioloalveolar epithelium, with staining of both basal cells of the airways as well as areas of bronchiolar and squamous metaplasia (33/33, 100\%). Bronchiolar metaplasia simulated the staining pattern of normal airways, with p63 immunoreactive cells confined to the basal layers while squamous metaplasia showed extension of positively stained cells through several layers of epithelium (Figure 1c, d).

Three of five cases of atypical adenomatous hyperplasia were nonimmunoreactive while two had strong immunoreactivity, throughout the atypical epithelium (Figure 1e, f). Nuclear immunoreactivity was seen in $4 / 33(12 \%)$ cases of adenocarcinomas while the remainder was negative (Figure 2a). Two cases showed $<1 \%$ and one case showed $5 \%$ positivity of malignant cells. One case, showing less than $1 \%$ immunoreactivity, was a percutaneous biopsy, with predominantly reactive alveolar epithelium adjacent to minimal amount of neoplastic tissue. In this case, distinction of malignant from reactive epithelium was difficult based exclusively on histologic criteria. In the open lung 

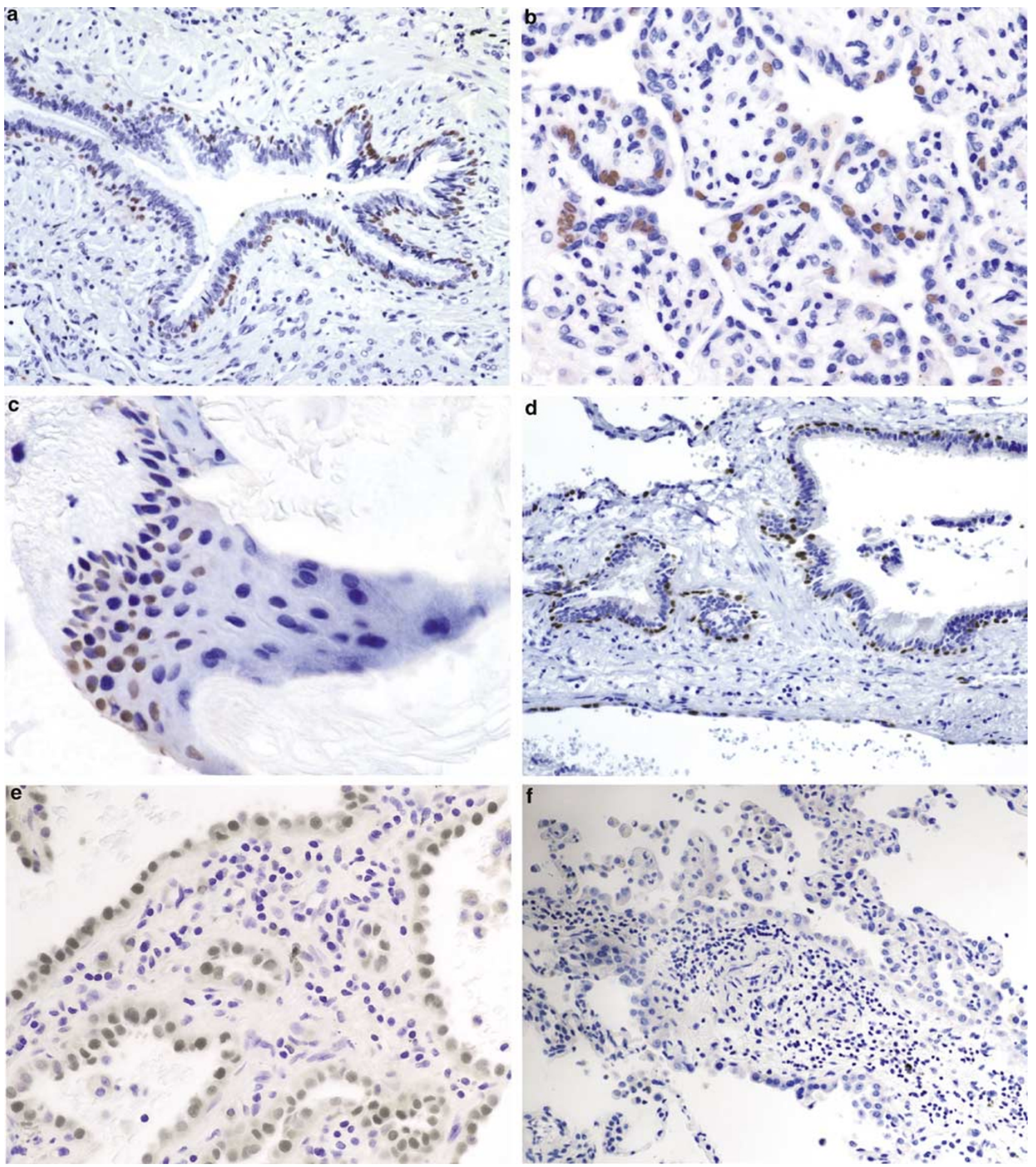

Figure 1 p63 immunoreactivity in reactive/hyperplastic lung: (a) normal bronchiole, $\times 20$; (b) organizing pneumonia, $\times 40$; (c) squamous metaplasia, $\times 40$; (d) bronchiolar metaplasia, $\times 20$; (e) atypical adenomatous hyperplasia $(80 \%$ positivity), $\times 40$; (f) atypical adenomatous hyperplasia, negative, $\times 20$.

biopsies, one adenocarcinoma showed scattered immunoreactive neoplastic cells, while the other showed nuclear immunoreactivity at the advancing edge of the tumor, which was again histologically indistinguishable from staining of pre-existing bronchial reserve/alveolar duct cells (Figure 2b). One additional case of well-differentiated adenocarcinoma, initially diagnosed as pulmonary scar with alveolar epithelial hyperplasia and mild atypia, showed $80 \%$ positivity of malignant cells 


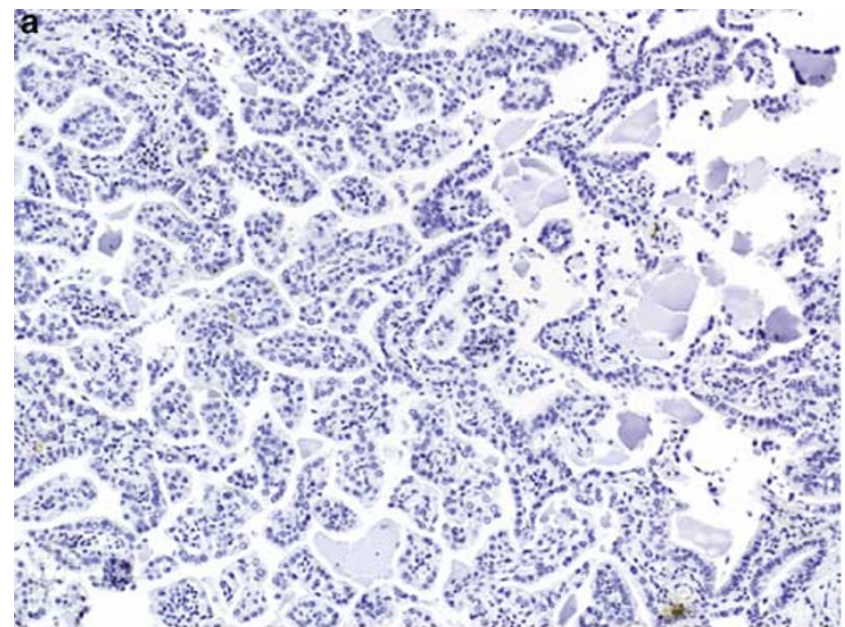

$b$,
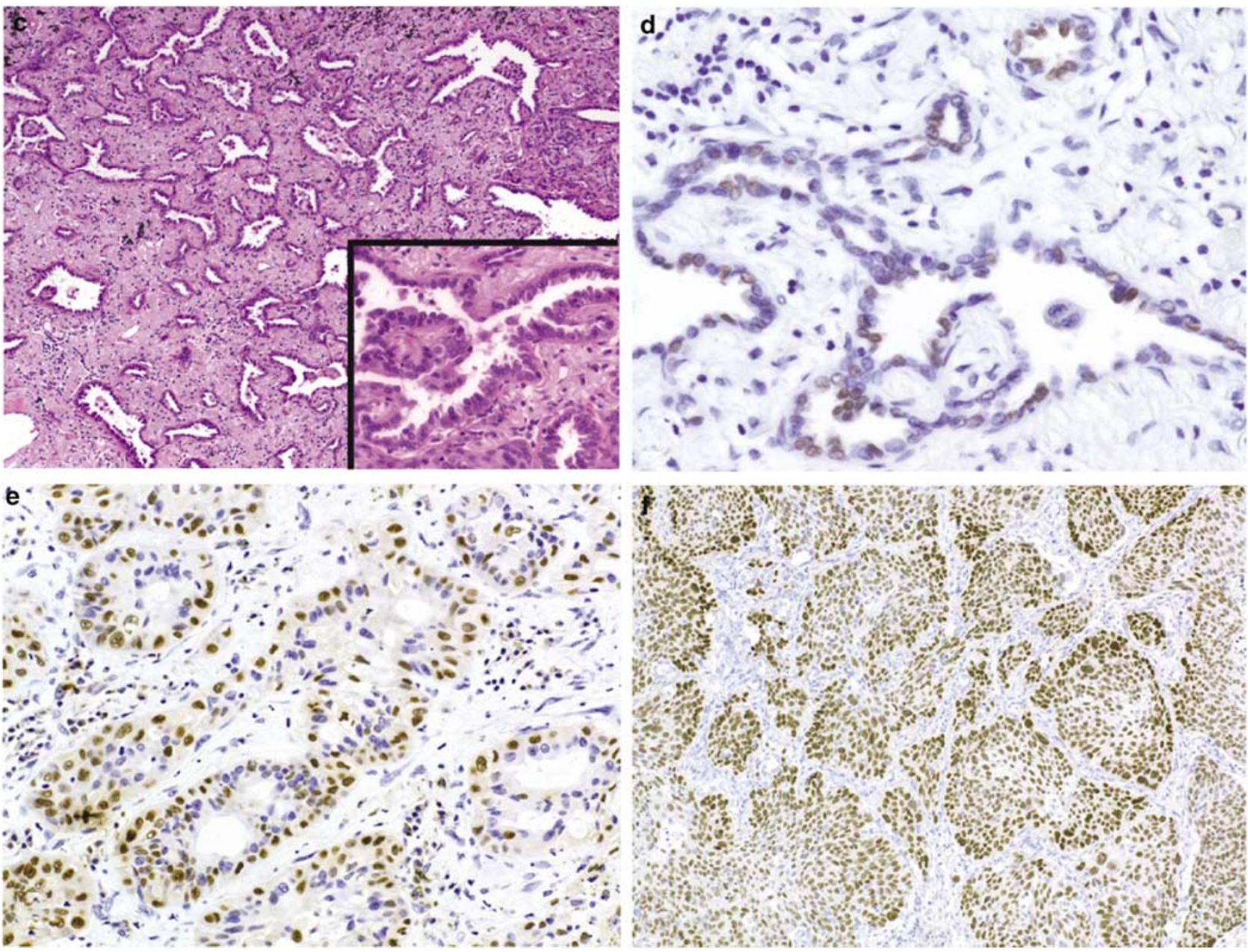

Figure 2 p63 immunoreactivity in pulmonary neoplasms: (a) papillary adenocarcinoma, nonreactive, $\times 10$; (b) adenocarcinoma with few immunoreactive cells $(1 \%)$ at advancing edge, $\times 20 ;(\mathbf{c}-\mathbf{d})$ adenocarcinoma with numerous immunoreactive cells $(80 \%), \times 10$ and $\times 40$ (H\&E and immunostain); (e) adenosquamous carcinoma, glandular component, $\times 20$; (f) squamous cell carcinoma, extensive immunoreactivity $(90 \%), \times 10$.

(Figure 2c, d). In rare instances, adenocarcinomas were noted to have retrograde spread up the bronchial tree. In these cases, scattered positive reserve cells could be identified. Comparison of p63 immunostaining of reactive and neoplastic prolif- erations on transbronchial biopsy specimens is shown in Figure 3.

All cases of adenosquamous carcinomas were immunoreactive (5/5). Immunoreactivity in the squamoid areas ranged from focal (5\%) to extensive 

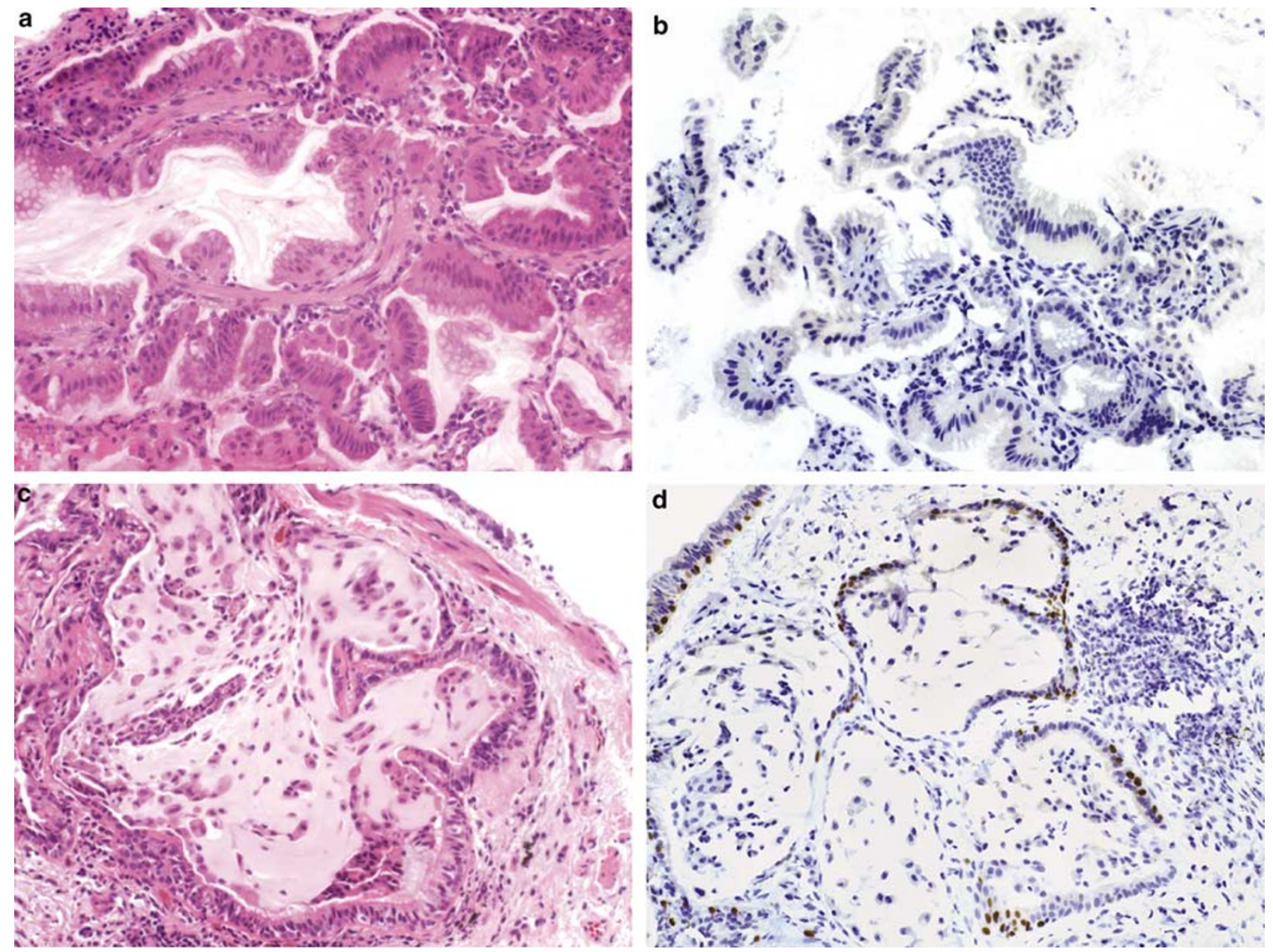

Figure 3 Transbronchial biopsy: (a) pulmonary adenocarcinoma, $\times 40$, H\&E; (b) pulmonary adenocarcinoma, $\times 20$, p63 immunostain; (c) usual interstitial pneumonia, $\times 20$, H\&E; (d) usual interstitial pneumonia, $\times 20$, p63 immunostain.

Table 1 Diagnostic categories and p63 immunoreactivity

\begin{tabular}{lccc}
\hline Diagnosis & No. of cases & $\begin{array}{c}\text { No. of cases with p63 } \\
\text { immunoreactivity (\%) }\end{array}$ & Range of p63 immunoreactivity \\
\hline Diffuse alveolar damage & 5 & $5(100)$ & N/A \\
Cryptogenic organizing pneumonia & 5 & $5(100)$ & N/A \\
Honeycomb lung & 23 & $23(100)$ & N/A \\
Apical cap & 10 & $10(100)$ & N/A \\
Atypical adenomatous hyperplasia & 5 & $2(40)$ & $80-100 \%$ \\
Adenocarcinoma & 33 & $5(10)$ & $<1-80 \%$ \\
Adenosquamous carcinoma & 5 & $3(100)$ & Squamoid areas: 5-90\% \\
Squamous carcinomalar areas: neg-20\% & & $60-90 \%$ \\
\hline
\end{tabular}

$(90 \%)$, with majority showing $>50 \%$ positivity of tumor cells. The adenocarcinomatous areas showed a basilar pattern of staining in $20 \%$ of tumor cells in three cases (Figure 2e). The three cases of squamous carcinomas showed more widespread p63-immunoreactivity compared to their pure glandular counterparts, with percentage of immunoreactive malignant cells ranging between 60 and 90\% (Figure 2f).
Results of p63 immunostaining in the various diagnostic categories are summarized in Table 1.

\section{Discussion}

In this study, we assessed the usefulness of p63 immunostain in distinguishing reactive and 
neoplastic glandular proliferations of the bronchioloalveloar epithelium.

p63 shares structural homology with p53, but unlike this tumor suppressor gene with welldescribed antiapoptotic functions, the role of p63 is less well understood. It is expressed in many stratified epithelia including skin, esophagus, larynx, cervix, and bladder but is confined to basal cells in glandular epithelium including breast, prostate and lung. ${ }^{6}$ Squamous cell carcinomas at these sites, however, express high levels of p63 protein, localized to the nucleus. ${ }^{6}$

The different isoforms of p63 are thought to have different functions as well. The truncated forms are thought to inhibit cell cycle arrest and apoptosis driven by transactivating p63/p53 interaction. ${ }^{12}$ The truncated isoforms are preferentially expressed in the basal cell compartment of normal epithelium ${ }^{12,13}$ and transactivating forms are more widely distributed in benign and neoplastic epithelium. ${ }^{14}$ Consequently, different isoforms of p63 appear to play a role in maintaining the epithelial stem cell population, spurring epithelial differentiation and inducing neoplasia. ${ }^{14}$ This is evidenced by the fact that p63 knockout mice fail to develop skin, hair, mammary tissue, prostrate, salivary glands, and have striking changes in other epithelia including urogenital tract and gastric mucosa. ${ }^{15,16}$ Interestingly, no pulmonary abnormalities have been described in the absence of p63 protein in these mouse models. This observation has been attributed to the slower rate of bronchial stem cell division (and depletion), compared to other epithelia.

Differentiating well-differentiated adenocarcinomas from benign bronchioloalveolar proliferation with reactive atypia is difficult, especially on small biopsy specimens. ${ }^{2}$ Conceptually, the situation may be compared to prostate needle biopsies, where basal cell markers are a useful adjunct to pathologic diagnosis. In the prostate, p63 has been shown to have an equal $^{8}$ or greater sensitivity than high molecular cytokeratin ${ }^{7,9}$ and a $100 \%$ specificity. $^{9}$ Shah et $a l^{9}$ demonstrated that p63 showed a higher percentage of positive staining basal cells in $41 \%$ of prostate needle biopsies compared with highmolecular-weight cytokeratin and offered advantage in $7 \%$ of diagnostically challenging cases. Interestingly, there were no significant differences in staining of benign glands using p63 alone when compared with a combined p63/high-molecularweight cytokeratin cocktail. ${ }^{7}$ These studies highlight the robustness of p63 as a basal cell marker in the prostate.

In the lung, p63 expression has been studied in various neoplasms as a prognostic indicator. It is most frequently expressed in squamous cell carcinomas where it shows strong staining of peripheral 'basilar' cells in well-differentiated variants and widespread staining in less differentiated forms. ${ }^{11}$ Adenocarcinomas are less frequently positive, with nuclear staining present in $15 \%$ of cases in one study $^{10}$ and $63 \%$ of cases in another. ${ }^{11}$ In the latter study, however, areas of lepidic growth of bronchioloalveolar carcinoma (comprising seven of 30 cases of adenocarcinomas studied) over existing alveolar architecture were scored as positive, and this may account for the higher proportion of p63positive adenocarcinoma cases. In both studies, p63 immunoreactivity was described as rare, focal or multifocal, sharply contrasting with larger, positively stained areas of squamous cell carcinoma. The mean percentage of positively stained cells in adenocarcinomas was approximately $5 \% .{ }^{10}$

Our results show fewer cases of p63 immunoreactive adenocarcinomas $(4 / 33,12 \%)$. In some cases, entrapped benign epithelium at the edge of tumor showed nuclear staining. Histologically, many of these glandular elements were easily distinguishable from the adjacent adenocarcinomas and were therefore considered negative. In two cases, however, atypical cells at the periphery of the tumor that showed staining, were difficult to unequivocally differentiate from lepidic growth of tumor over normal alveolar architecture and retrograde spread of adenocarcinoma into small airways. These cases were considered positive. It is clear that p63 is of limited use when biopsies are taken exclusively from the junction of normal and neoplastic lung.

Atypical adenomatous hyperplasia is often an incidental finding, encountered in resection specimens rather than in smaller biopsy material. ${ }^{17}$ However, high-resolution CT has increased the detection rate of nodules in the peripheral lung less than $1 \mathrm{~cm}$ in size that are then excised via videoassisted thoracoscopic surgery or open-lung biopsy. ${ }^{17,18}$ In one study, atypical adenomatous hyperplasia was the cause of radiologic findings in $18 \%$ of open lung biopsies, and pathologic diagnosis was based on morphology alone. ${ }^{18}$ Absence of p63 immunoreactivity in the majority of cases that we studied suggests that this marker has limited utility in distinguishing carcinoma from atypical adenomatous hyperplasia. From the standpoint of pathogenesis, it appears that the reserve cell phenotype is variably maintained in these preneoplastic proliferations. However, given the small numbers of cases, our results should be interpreted with caution.

One case of adenocarcinoma, which showed the greatest p63 immunoreactivity (80\%), was interesting in that it was a difficult diagnosis histologically and in fact, was initially misdiagnosed as alveolar epithelial hyperplasia in a pulmonary scar. The histologic resemblance to a scar together with the widespread p63 positivity in malignant cells in this case, suggests the evolution of peripheral adenocarcinoma through epithelial hyperplasia within a scar or that some adenocarcinomas maintain the reserve cell phenotype.

Fibrotic lung processes whether focal as in apical caps or generalized as in usual interstitial pneumonia, showed staining of basal cells of the 
bronchioloalveolar tree, increasing in areas of squamous and bronchiolar metaplasia. The results are similar to those previously reported in the normal lung. ${ }^{10,11}$ Pelosi et al, ${ }^{10}$ also demonstrated by double labeling with Ki67, that p63-immunoreactivity was confined to the Ki67-positive proliferating compartment of the respiratory epithelia. It seems reasonable to assume that these cells would increase in number, in long-standing reactive conditions of the lung. Unlike the normal lung, therefore, scattered alveolar epithelial cells also showed p63 immunoreactivity in cases of pulmonary scarring. p63 immunoreactivity, however, was less uniform in acute/subacute lung conditions, suggesting that there is a lag time between epithelial injury and the reparative response of the basal cells.

From a practical standpoint, one problem that we encountered using the p63 antibody, was nonspecific cytoplasmic staining of some mucinous adenocarcinomas, and inflammatory cells in cases of usual interstitial pneumonia. This problem was attributed to increased antigenicity secondary to microwave treatment and was resolved by decreasing the duration of this treatment and by optimizing the dilution for our formalin-fixed specimens, using similarly fixed in-house prostate controls.

In summary, our results highlight the differential staining pattern of p63 across the spectrum of bronchioloalveolar proliferations of the lung. The sensitivity and specificity of this marker for differentiating reactive from neoplastic proliferations is maintained even in small biopsy specimens. Although the marker does not reliably differentiate atypical adenomatous hyperplasia from adenocarcinoma, the small size of the former lesion makes it less amenable to percutaneous/transbronchial biopsy. While the role of p63 in oncogenesis remains to be elucidated, in the day-to-day practice of pathology, p63 may serve as a useful marker in diagnostically difficult cases of glandular proliferations of the lung.

\section{References}

1 Brambilla E, Travis WD, Colby TV, et al. The new World Health Organization classification of lung tumours. Eur Respir J 2001;18:1059-1068.

2 Colby TV. Malignancies in the lung and pleura mimicking benign processes. Semin Diagn Pathol 1995;12:30-44.

3 Huang MS, Colby TV, Therneau TM, et al. DNA ploidy and protein content in bronchioloalveolar carcinoma multi-variable flow cytometry. Cytometry 1996;26: 253-259.

4 Kodama T, Biyajima S, Watanabe S, et al. Morphometric study of adenocarcinomas and hyperplastic epithelial lesions in the peripheral lung. Am J Clin Pathol 1986;85:146-151.

5 Kitamura H, Kameda Y, Nakamura N, et al. Atypical adenomatous hyperplasia and bronchoalveolar lung carcinoma. Analysis by morphometry and the expressions of p53 and carcinoembryonic antigen. Am J Surg Pathol 1996;20:553-562.

6 Di Como CJ, Urist MJ, Babayan I, et al. p63 expression profiles in human normal and tumor tissues. Clin Cancer Res 2002;8:494-501.

7 Zhou M, Shah R, Shen R, et al. Basal cell cocktail (34betaE12 + p63) improves the detection of prostate basal cells. Am J Surg Pathol 2003;27:365-371.

8 Weinstein MH, Signoretti S, Loda M. Diagnostic utility of immunohistochemical staining for p63, a sensitive marker of prostatic basal cells. Mod Pathol 2002;15: 1302-1308.

9 Shah RB, Zhou M, LeBlanc M, et al. Comparison of the basal cell-specific markers, 34betaE12 and p63, in the diagnosis of prostate cancer. Am J Surg Pathol 2002;26: 1161-1168.

10 Pelosi G, Pasini F, Olsen Stenholm C, et al. p63 immunoreactivity in lung cancer: yet another player in the development of squamous cell carcinomas? J Pathol 2002;198:100-109.

11 Wang BY, Gil J, Kaufman D, et al. p63 in pulmonary epithelium, pulmonary squamous neoplasms, and other pulmonary tumors. Hum Pathol 2002;33: 921-926.

12 Yang A, Kaghad M, Wang Y, et al. p63, a p53 homolog at 3q27-29, encodes multiple products with transactivating, death-inducing, and dominant-negative activities. Mol Cell 1998;2:305-316.

13 Signoretti S, Waltregny D, Dilks J, et al. p63 is a prostate basal cell marker and is required for prostate development. Am J Pathol 2000;157:1769-1775.

14 Nylander K, Vojtesek B, Nenutil R, et al. Differential expression of p63 isoforms in normal tissues and neoplastic cells. J Pathol 2002;198:417-427.

15 Yang A, Schweitzer R, Sun D, et al. p63 is essential for regenerative proliferation in limb, craniofacial and epithelial development. Nature 1999;398:714-718.

16 Mills AA, Zheng B, Wang XJ, et al. p63 is a p53 homologue required for limb and epidermal morphogenesis. Nature 1999;398:708-713.

17 Ohtsuka T, Nomori $\mathrm{H}$, Horio $\mathrm{H}$, et al. Radiological examination for peripheral lung cancers and benign nodules less than $10 \mathrm{~mm}$. Lung Cancer 2003;42: 291-296.

18 Kishi K, Homma S, Kurosaki A, et al. Small lung tumors with the size of $1 \mathrm{~cm}$ or less in diameter: clinical, radiological, and histopathological characteristics. Lung Cancer 2004;44:43-51. 